\title{
Coordinated Mechanical Loads and Power Optimization of Wind Energy Conversion Systems with Variable-weight Model Predictive Control Strategy
}

Lin, Zhongwei; Chen, Zhenyu ; Liu, Jizhen; Wu, Qiuwei

Published in:

Applied Energy

Link to article, DOI:

10.1016/j.apenergy.2018.11.089

Publication date:

2018

Document Version

Early version, also known as pre-print

Link back to DTU Orbit

Citation (APA):

Lin, Z., Chen, Z., Liu, J., \& Wu, Q. (2018). Coordinated Mechanical Loads and Power Optimization of Wind

Energy Conversion Systems with Variable-weight Model Predictive Control Strategy. Applied Energy, 236, 307317. https://doi.org/10.1016/j.apenergy.2018.11.089

\section{General rights}

Copyright and moral rights for the publications made accessible in the public portal are retained by the authors and/or other copyright owners and it is a condition of accessing publications that users recognise and abide by the legal requirements associated with these rights.

- Users may download and print one copy of any publication from the public portal for the purpose of private study or research.

- You may not further distribute the material or use it for any profit-making activity or commercial gain

- You may freely distribute the URL identifying the publication in the public portal 


\title{
Coordinated Mechanical Loads and Power Optimization of Wind Energy Conversion Systems with Variable-weight Model Predictive Control Strategy
}

\author{
Zhongwei Lin ${ }^{\mathrm{a}}$, Zhenyu Chen ${ }^{\mathrm{a}, *}$, Jizhen $\mathrm{Liu}^{\mathrm{a}}$, Qiuwei $\mathrm{Wu}^{\mathrm{b}}$ \\ ${ }^{a}$ State Key Laboratory of Alternate Electrical Power System with Renewable Energy Sources, \\ School of Control and Computer Engineering, \\ North China Electric Power University, Beijing 102206, P. R. China \\ ${ }^{b}$ Center for Electric Power and Energy, Department of Electrical Engineering, \\ Technical University of Denmark, Kgs. Lyngby 2800, Denmark
}

\begin{abstract}
For wind energy conversion systems operating above the rated wind speed, the frequent pitch actions regulate the mechanical power as the rated one with the cost of blade and drive shaft loads. It is meaningful to maintain the desired power with appropriate pitch sensitivity related to the wind speed fluctuations, which can further reduce the mechanical loads of wind turbines with a longer service life. To quantify the blade pitch sensitivity, the blade pitch standard deviation is introduced to connect the pitch actions with the blade and drive shaft loads. Within a variable-weight model predictive control (MPC) strategy, both generator power output quality and load conditions are optimized through the pitch/torque participation coordination based on the Pareto analysis. Moreover, the MPC-weight matrix could be updated adaptively through the wind status assessment. The comparisons between the proposed strategy and the traditional gain scheduling PI one show the effectiveness. Several suggestions are also concluded for industrial wind turbines with MPC implementations.
\end{abstract}

\footnotetext{
Whis work was partially supported by the Research on intelligent control technology of wind turbine of China Guodian United Power Technology Co., Ltd. (No. GPOD17001), the Development and application of key technologies for grid-friendly intelligent wind farm energy management system of Guodian New Energy Technology Research Institute Co., Ltd. (No. GJNY-18-22), the National Natural Science Foundation of China (No. U1766204), the Fundamental Research Funds for the Central Universities (No. 2018ZD05, 2018BJ0116).

*Corresponding author

Email address: czy_ac@ncepu.edu.cn (Zhenyu Chen)
} 
Keywords: Wind energy conversion system, Coordination, Pareto, Optimization

\section{Introduction}

In recent years, wind energy has been growing rapidly due to the ever-increasing energy demand and quest of mitigating the climate change. According to the GWEC Global Wind Energy Report 2017 [1], the wind power installations was 52.5 GW in 2017, bringing the global total to $539 \mathrm{GW}$. The wind energy is captured and converted by wind energy conversion systems (WECSs). As the main mechanism of wind power production, the WECS is usually operated in four wind speed regions, i.e., two shutdown regions above the cut-out wind speed or below the cut-in wind speed, and two operating regions to maximize wind energy conversion efficiency below the rated wind speed $[2,3,4]$, and to maintain the output power at the rated power above the rated wind speed $[5,6]$. Several researchers have discussed the transition strategy between the below and above rated speed regions to achieve smooth transition or stabilizing output [7]. The mechanical power captured by the wind turbine can be expressed as a static nonlinear mapping of the blade pitch angle and tip speed ratio as

$$
\begin{gathered}
P_{a}=\frac{1}{2} \rho \pi R^{2} v^{3} C_{p}(\beta, \lambda), \\
\lambda=\frac{R \omega_{r}}{v} .
\end{gathered}
$$

In (1), $P_{a}$ is the aerodynamic power, $\beta$ is the collective pitch angle, $\omega_{r}$ is the angular velocity of the rotor, $v$ is the wind speed, $\rho$ is the air density and $R$ is the rotor radius. The tip speed ratio $\lambda$ is defined as in (2). Detailed WECS models can be found in $[2,8,9]$. The wind power coefficient $C_{p}$ reflects the wind energy capture capability of the wind turbine, which is a nonlinear function of the blade pitch angle and tip speed ratio. For different wind turbines, the polynomial expressions of $C_{p}$ are usually with a similar form but different parameters. The adopted wind energy coefficient for the simulated wind turbine can be referred to [10].

Due to the non-linearity of WECS aerodynamics, many works have applied the nonlinear dynamic theory to improve the wind turbine design and operations. Ref.[11] has combined the mechanical side and electrical side including the generator and bidirectional converter as the WECS model. With a receding-horizon adaptivesliding-mode strategy, both modeling uncertainties and disturbances have been suppressed for WECS. An adaptive back-stepping pitch reference was proposed in [12] to improve the WECS power output and drive-train torque performances. [13] has designed a novel robust sliding-mode control using nonlinear perturbation observers 
for WECS. A nonlinear regulation strategy was proposed in [14] to improve the dynamic characteristic of WECS. The WECS is linearized by the feedback linearization method, then the regional pole placement technique is applied to regulate the dynamic response. With the development of artificial intelligence, advanced intelligent algorithms also play important roles in wind energy research field [15]. Ref.[16] reviewd the theory of inventive problem solution in wind turbine innovative design, which shows a systematic methodology to enhance the capability of developing innovative products and overcomes the main design problems. An adaptive neuro-fuzzy inference system was introduced in [17] to estimate the wind farm power production, cost and efficiency with the increasing number of wind turbines. Intelligence algorithms can also be applied in the WECS operations with a better power tracking ability [18, 19]. [20] introduced a multi-objective particle swarm optimization-based method to optimize yaw control parameters for higher achievable power extraction efficiency.

On the other hand, the linear state-space model is usually adopted for WECS based on the operating-point linearization, which can better represent the relations between the system state, input and output variables. Based on the operating-point linearized model, all poles could be calculated to reflect the system stability and dynamic performance. Many linear wind turbine operation and control strategies have been proposed. Ref.[21] proposed an extended state-space model with the stochastic characteristic of wind velocity turbulence, which guarantees the dynamic performance by the regional pole placement. [22] focused on the transient analysis of wind turbines considering wind speed disturbances and a pitch control malfunction. Meanwhile, the operation or control problem could be formulated as an optimization one. The optimal variables are obtained by minimizing the objective function, such as robust control, linear quadratic control and model predictive control (MPC) [23, 24]. Ref.[25] and [26] proposed methods to establish the control problem and optimize the weight matrix in the MPC framework. By using the Pareto theory to qualitatively analyze two objectives, a satisfactory solution could be obtained by calculating the gradient. This concept inspires our study on further analyzing the Pareto characteristic between optimization objectives to develop the coordinated controllers of wind turbines.

From the WECS operation point of view, the blade pitch and generator torque are working together to stabilize the power output above the rated wind speed. For the traditional strategies, the generator torque is usually set as inversely proportional to the generator speed, while the blade pitch action is the dominant actuator to keep the constant power output. It is potential to make full use of the generator torque loop to work with the blade pitch action coordinately. [27] has analyzed the 
effect of fixed pitch angle on the loads and moments as well as the aerodynamics of wind turbines. A coordination strategy was proposed in [9] through the Pareto optimization theory, while the controller participation is adjusted by the pole placement technique. Due to the actuator limiters or steady state errors, it is still difficult to implement the proposed state-feedback control for the industrial wind turbine. Meanwhile, the MPC strategy deals with the control problem in the optimization framework, which has the advantage of handling actuator constraints and multiple objectives. However, the weight matrices of MPC affect the control performance directly. It is important to design the weight matrices if the MPC strategy is applied on the industrail wind turbines. Besides, as to the operation objectives, the wind turbine needs to be optimized for both output stability and load conditions. There are many load parameters, which have different causes and action modes. It is difficult to incorporate the load parameters into the optimization problem, which is often chosen subjectively [28]. In such a case, how to find an appropriate load performance index, and establish connections with the weight matrix for wind turbine optimization is the important prerequisite to implement the MPC strategy on industrial WECSs.

As the main contribution of this paper, the blade pitch sensitivity is analyzed to optimize the WECS operations above the rated wind speed, where a variable-weight MPC strategy is proposed with a new characteristic parameter connected to the load conditions. Both generator torque and blade pitch angle are regulated to reduce the load conditions while stabilizing the output power. Firstly, the coordination between controllers is analyzed through the adjustment of the MPC control-weight matrix. A characteristic parameter is introduced through the correlation analysis to represent both drive shaft load and blade load, i.e., the blade pitch standard deviation. Then the power output and load conditions are considered to find a satisfactory interval of control-weight matrix parameters. Subsequently, the model predictive controller is set to update the weight parameters dynamically corresponding to the wind status assessment. Based on these analysis, several MPC-weight-matrix tuning suggestions are summarized for further implementation on industrial WECS.

The rest of this paper is organized as follows: In Section 2, the MPC framework is presented for WECS. In Section 3, the blade-shaft loads characteristic parameter is proposed and analyzed. Section 4 investigates the multi-objective optimization problem with the controller-coordination based on Pareto optimal theory. Section 5 introduces the wind-assessment-based variable-weight strategy and analyzes the effectiveness. Several practical engineering suggestions are listed in Section 6. Section 7 concludes this paper. 


\section{MPC Strategy of WECSs}

In this paper, the WECS model and parameters are referred to the 5-MW horizontal axis wind turbine (HAWT) provided in FAST (Fatigue, Aerodynamics, Structures, and Turbulence). The FAST code is a comprehensive aeroelastic simulator, which is capable of predicting both the extreme and fatigue loads of two- and threebladed HAWTs. Related information about FAST and the adopted wind turbine can be obtained from $[29,30]$. In this section, the MPC strategy for the time-constant linear wind turbine model is introduced, which transforms the control problem into a standard quadratic programming (QP) one. The optimization solver is the commercial optimization software Mosek. Related information about the optimization software can be referred to [31].

Firstly, the nonlinear wind turbine with the flexible drive-shaft is linearized into a three-order linear state-space model, where two control inputs (the references of generator torque and blade pitch angle) are considered in the same framework. The detailed linearization process and the wind turbine state-space model can be referred to [9]. Due to the strong effectiveness of MPC for industrial application, it is natural to discuss the MPC method for WECSs [7, 25, 26]. Should be noticed that, a three-order model is used to model the wind turbine for controller design, while the higher-order model is useful to test with full consideration of all components in the wind turbine. Since the model-based prediction is required during the MPC design, the high-order model will increase the optimization order and require more time to achieve any optimal solution. The three-order model could reach an acceptable balance between the wind turbine modeling and the optimization order, which is adopted for the MPC design.

\subsection{Optimization Functions}

For the linearized WECS model, the state variables contain the shaft equivalently torsional angle, rotor speed and generator speed: $x=\left[\delta, \omega_{r}, \omega_{g}\right]^{\prime}$, and the control inputs are the references of the blade pitch angle and the generator torque: $u=$ $\left[\beta_{r e f}, T_{e m}^{r e f}\right]^{\prime}$. The continuous state-space model is written as: $\dot{x}=A_{c} x+B_{c} u$, which can be dispersed into a discrete system: $x(k+1)=A x(k)+B u(k)$ through the zero-order holder method. Set the initial time as $k$, the prediction horizon as $N$, then the predictive system can be organized as,

$$
X(k)=F_{x} x(k)+G_{x} U(k),
$$


in which

$$
\begin{gathered}
X(k)=\left[\begin{array}{c}
x(k+1) \\
\vdots \\
x(k+N)
\end{array}\right], U(k)=\left[\begin{array}{c}
u(k) \\
\vdots \\
u(k+N-1)
\end{array}\right] \\
F_{x}=\left[\begin{array}{c}
A \\
\vdots \\
A^{N}
\end{array}\right], G_{x}=\left[\begin{array}{ccc}
B & \cdots & 0 \\
\vdots & \ddots & 0 \\
A^{N-1} B & \cdots & B
\end{array}\right]
\end{gathered}
$$

The optimization problem related to MPC can be expressed as follows: For each control step $k$, find the control parameters $[u(k), u(k+1), \cdots, u(k+N-1)]$ by solving the QP problem. Take the first step control $u(k)$ to sustain the defined optimization objective and constrains, which are related to the system performance in the next $N$ steps. Then repeat the previous process for the next step. The optimization function is written as:

$$
\min _{U(k)} J(k)=\|X(k)\|_{Q_{x}}^{2}+\|U(k)\|_{R_{u}}^{2}
$$

where $Q_{x}$ and $R_{u}$ are the state and control weight matrices respectively. Set

$$
H=2\left(G_{x}^{T} Q_{x} G_{x}+R_{u}\right)
$$

and

$$
f=2\left(F_{x} x(k)\right)^{T} Q_{x} G_{x},
$$

then the optimization problem in MPC could be converted into a standard QP problem as,

$$
\min _{U(k)} J(k)=\frac{1}{2} U(k)^{T} H U(k)+f U(k) .
$$

Obviously, the weight matrix has a closed relationship with the controller design and practical effectiveness.

\subsection{Constraints}

The following constraints are used in the MPC design of WECS. Firstly, the constraints of state variables are given as,

$$
\left\{\begin{array}{l}
\omega_{g} \leq 1.1 \cdot \omega_{g_{\text {Rated }}} \\
\omega_{r} \leq 1.1 \cdot \omega_{r_{\text {Rated }}}
\end{array}\right.
$$

in which $\omega_{g}$ and $\omega_{r}$ are the generator speed and rotor speed. The speed symbols with "Rated" are the optimal speed at rated conditions. These constraints keep the speed within a fixed band around the rated speed for the safety consideration. 
Besides, the constraints related to control parameters are also considered:

$$
\left\{\begin{array}{l}
-8^{\circ} / s \leq \delta \beta_{\text {ref }} \leq 8^{\circ} / \mathrm{s} \\
0^{\circ} \leq \beta_{\text {ref }} \leq 90^{\circ} \\
T_{e m}^{r e f} \leq T_{e m}^{\text {Rated }} \cdot 110 \%
\end{array}\right.
$$

The blade pitch angle is set between $0^{\circ}$ and $90^{\circ}$, and the pitch action is limited to $8^{\circ} / \mathrm{s}$ in the absolute value to avoid the stalling. The generator torque maximum value is set to $10 \%$ above the rated value to avoid any excessive overloading.

\subsection{Weight Matrices}

For the optimization problem (4) of the MPC, the weight matrix is the key to connect the optimization function to the desired control effectiveness, which describes the weight of state variables and the coordination of control inputs. In this paper, the wind turbine control is realized by adjusting the control input participation through the control-weight-matrix adjustment. The state-weight matrix $Q_{x}$ is tuned previously and kept constant during the adjustment process of $R_{u}$. Considering the convenience of dimensions and further optimization feasibility, $Q_{x}$ and $R_{u}$ are proposed with the following structures:

$$
Q_{x}=\left[\begin{array}{ccc}
1 & 0 & 0 \\
0 & 10^{6} & 0 \\
0 & 0 & 10^{2}
\end{array}\right], R_{u}=\left[\begin{array}{cc}
a & 0 \\
0 & 10^{b}
\end{array}\right]
$$

Two parameters on the diagonal of the control-weight matrix $R_{u}$ are the weights for each control input respectively, which coordinates the two controllers. Considering the two control inputs are with different order of magnitudes, the generator torque weight is set to the form of $10^{b}$ to alleviate the sensitivity difference of input parameters to weight coefficients, and $a$ is the blade pitch angle weight. Both weight factors are adjusted in different simulations to obtain the responses for further coordination optimizations.

\section{Analysis of the Load Characteristic Parameter}

Traditional MPC for WECS mitigates the torque fluctuation by the fatigue loads reduction. However, there still exists an unclear gap. Herein, another characteristic parameter is introduced to further represent the fatigue loads for the optimal operations. Loads on the blade and the drive shaft are the main load parameters considered in this study. 


\subsection{Blade Loads Analysis}

The blade coordinate system is usually resolved into $x_{c}, y_{c}, z_{c}$ three directions. The origin of the blade cone coordinate system is staying on the focal point of the blade extension lines. The coordinate system for each blade subscript $i$ ranges 1,2 , or 3 (representing three blades respectively) and rotates with the rotor, which does not pitch with the blades and also teeters in two-bladed models. The $z_{c, i}$ axis is pointing along the pitch axis towards the tip of blade $i$. The $y_{c, i}$ axis is pointing towards the trailing edge of blade $i$ if the pitch and twist were zero and parallel with the chord line. The $x_{c, i}$ axis is orthogonal with the $y_{c, i}$ and $z_{c, i}$ axes such that they form a right-handed coordinate system. The blades bending moment on three axis are marked as follows: RootMxc (in-plane moment on the $x_{c}$ axis), RootMyc (out-ofplane moment on the $y_{c}$ axis) and RootMzc (pitching moment on the $z_{c}$ axis). The left one of Figure 1 is the commonly used coordinate system. Should be noticed that, the coordinate system is usually established based on the downwind direction. During simulations, the wind comes from the downwind direction, and the wind turbine is also deflected to the downwind that faces the wind direction on the rotor surface. But the directions of the blade coordinate systems would not change their bending moments and directions. In order to maintain the consistency, the schematic diagram of the blade coordinate system selects the same viewing angle, and the windward and leeward directions of rotor surface are the only difference. The right one in Figure 1 is the actually used blade coordinate system in simulations that the turbine faces the downwind direction.
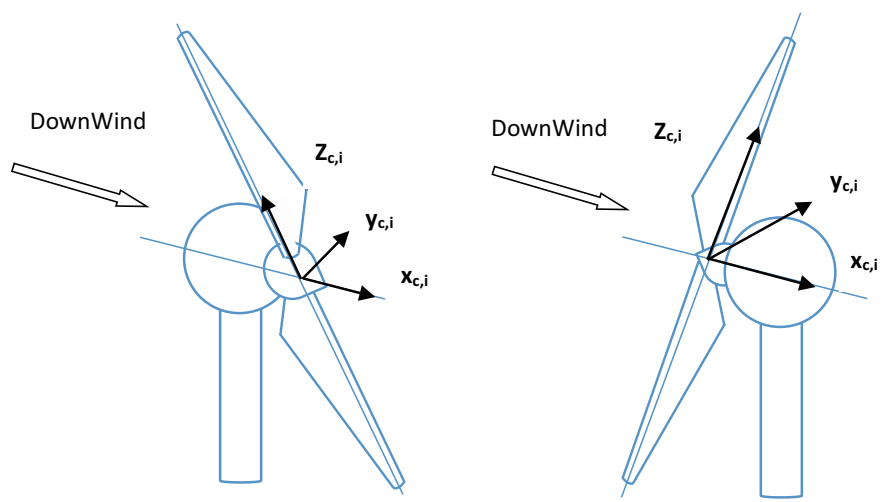

Figure 1: Blade Coordinate System

The three bending moments on the blade root are considered as the main parameters to represent the blade fatigue load conditions. Assuming that the value of a bending moment is positive, the mean value of the bending moment is closely 
related to the current wind speed. Obviously, the increase of blade pitch angle can surely reduce the thrust from the wind speed acting on the rotor surface, and reduce the blade root bending moment. But the consequence is a lower power capture than the rated power, which is not consistent with our objectives: to stabilize the power capture at the rated value while reducing the blade loads to extend the service life. It is unwise and unrealistic to reduce the blade load by increasing the blade angle without considering the power output. On the other hand, the bending moments are positively related to the mechanical stress, and once the mechanical stress exceeds the metal fatigue limit, the blade will be damaged. The greater the mechanical stress, or the more times the mechanical stress exceeding the fatigue limit, the greater the damage to the blades. The blade root loads responses based on a baseline controller provided in FAST is plotted as in Figure 2. Clearly, the in-plane moment on the $x_{c}$ axis changes from negative to positive and then turns around periodically. The out-of-plane moment on the $y_{c}$ axis is positive, and the pitching moment on the $z_{c}$ axis is negative. The above discussion also works in a similar way for the negative bending moments, which are still positive parameters but with an opposite direction. Hence, the three blade bending moments need to be analyzed with the maximum absolute value.
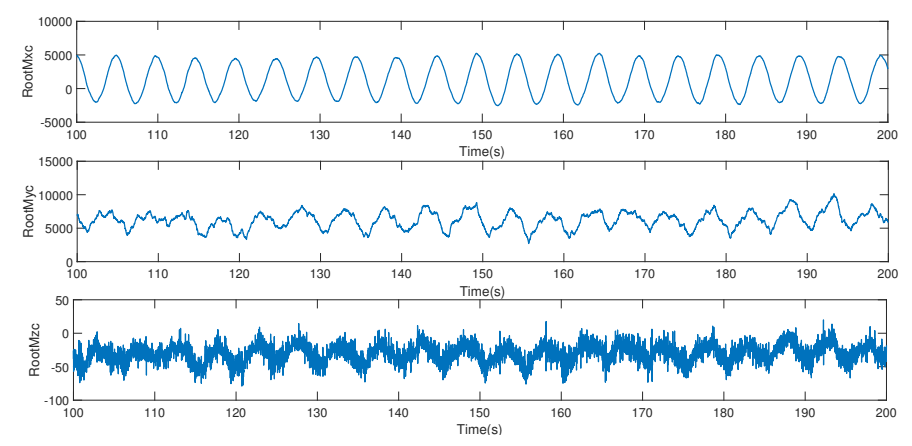

Figure 2: Blade Root Bending Moments under the Baseline Controller

Although load parameters like blade bending moments and shaft thrust forces can be easily obtained in FAST, they are still difficult to be qualified into the wind turbine control model. Once an appropriate parameter in the wind turbine model can be defined to characterize the blade loads, the related parameter can be optimized with the weight matrix in the model predictive controller. Motivated by this concept, several parameters within the wind turbine state-space model (the model can be referred to [9]) are calculated and compared with the blade bending moment parameters, which aims to find the correlation that the blade loads can be 
easily considered into the controller design and optimization. The analyzed parameters are normalized by the corresponding parameters obtained from an industrial baseline controller for the specific 5-MW wind turbine as in [29]. The norm values are obtained by $\frac{\psi_{\text {Self }}}{\psi_{B C}}$, where $\psi_{\text {Self }}$ are the respective response parameters under the model predictive controller, and $\psi_{B C}$ are the respective response parameters under the baseline controller. The parameters correlation is shown in Figure 3.
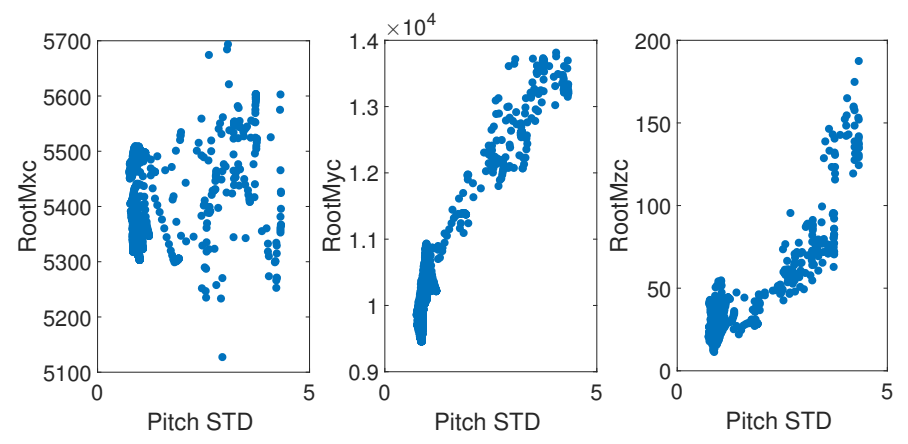

Figure 3: Blade Loads Parameter Correlation Analysis

In Figure 3, the $X$ axis is the blade pitch standard deviation (STD), and the $Y$ axes are the blade 1 root bending moments in $x c, y c, z c$ axes. It can be found that, these parameters are not positively related completely, but a higher pitch movement STD is always accompanied by higher bending moments in yc axis, and lower outof-plane bending moments can be achieved when the blade pitch movement STD is lower. Although the parameters correlations between the blade pitch STD and the bending moments in $x c, z c$ axes are unclear, the changes in both bending moments are very small compared to the out-of-plane bending moment, which can be ignored. Thus, the blade pitch movement STD can be used to evaluate the blade loads although it is not a strictly positive correlation, but a lower blade loads responses always can be guaranteed when the blade pitch movement STD is smaller.

\subsection{Shaft Loads Analysis}

Similarly as the blade coordinate system, the LSS (low speed shaft) coordinate system is also resolved into three directions $x_{s}, y_{s}, z_{s}$ and does not rotate with the rotor. But it does translate and rotate with the tower and yaws with the nacelle and furls with the rotor. The origin of the coordinate system is at the intersection of the $y_{s}-z_{s}$ plane and the rotor axis. The $x_{s}$ axis is pointing along the shaft in the nominally downwind direction. The $y_{s}$ axis is pointing to the left when looking from the tower toward the nominally downwind end of the nacelle. The $z_{s}$ axis 


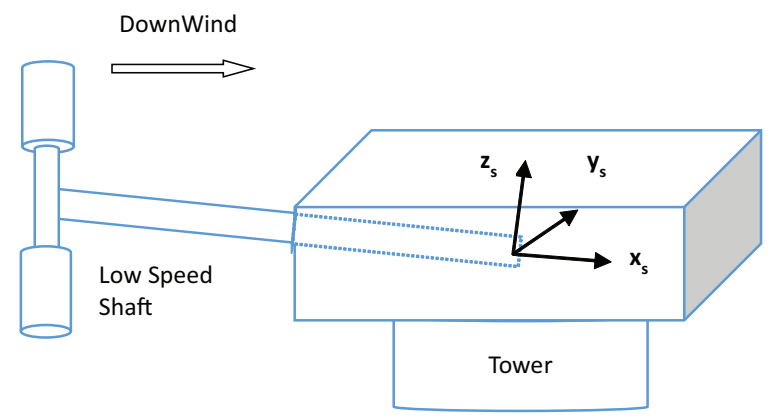

Figure 4: Shaft Coordinate System

is orthogonal with the $x_{s}$ and $y_{s}$ axes, such that three axes form a right-handed coordinate system. Similarly, the wind turbine faces the wind direction on the rotor surface during simulations. The LSS coordinate system is shown in Figure 4.

Shaft thrust forces on three axes are adopted to represent the low speed shaft (LSS) loads. Data analysis shows that, the LSS thrust forces are also related to the blade pitch movement STD. The blade pitch STD and LSS shaft thrust forces maximum absolute value are plotted in Figure 5. Clearly, the thrust force on the
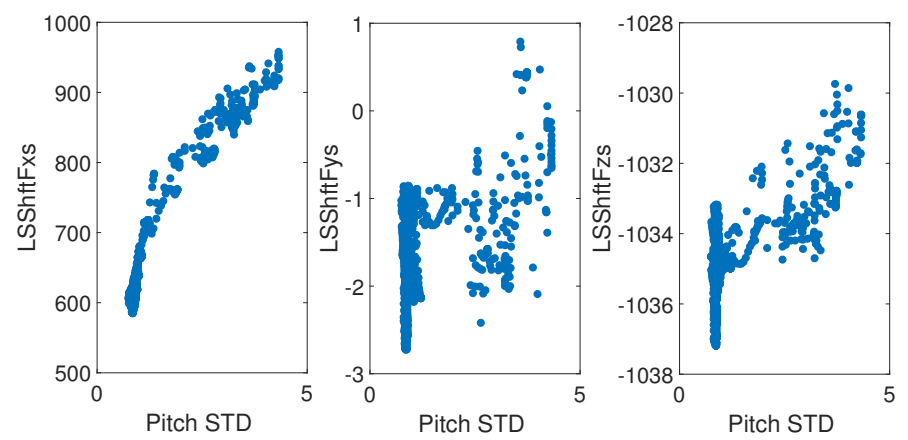

Figure 5: Shaft Loads Parameter Correlation Analysis

$x_{s}$ axis increases when the pitch STD increases. But the thrust force on the $y_{s}$ and $z_{s}$ axes shows different changes, which first declines rapidly and then increases with the increase of blade pitch STD. Fortunately, the thrust force on $y_{s}$ and $z_{s}$ axes are with the same trend and both of them are changed in a very small region less than 5 . The thrust force on the $x_{s}$ axis is the dominant element in all three thrust forces and can be reduced by decreasing the blade pitch actions STD. Therefore, the low speed 
shaft loads can be also connected with the blade pitch STD during optimizations: the lower the blade pitch STD, the smaller the drive shaft loads.

\section{Coordination Optimization}

In this section, the blade pitch movement STD is used to evaluate the blade and shaft loads based on the above discussions. Both the wind turbine mechanical loads and output quality are the parameters considered to optimize the wind turbine working conditions, and the control-input-weight matrix in the model predictive controller is used to evaluate the participation of each control parameters. The Pareto relationship is first analyzed based on the testing data. Several basic concepts of the Pareto theory and optimization theory can be referred to $[9,32]$.

During simulations, the wind turbine works under a turbulent wind condition with an effective wind speed of $15 \mathrm{~m} / \mathrm{s}$. Several simulations are carried out based on the model predictive control with different weight matrices. The weight factors are set as $a \in[0.2,8], b \in[-6,-2]$ based on the previous test. The response data is processed to calculate the generator power STD and blade pitch STD in order to evaluate the characteristics of output quality and wind turbine load conditions. In Figure 6 , the $x$ axis is the blade pitch movement STD and the $y$ axis is the generator power STD. The increase in $x$ axis means a worse situation in blade and shaft loads, and the increase in $y$ axis means a worse generator power stability condition. All dots of one curve are with the same blade pitch weight, and curves with different colors are corresponding to the specific responses with different blade pitch weights.

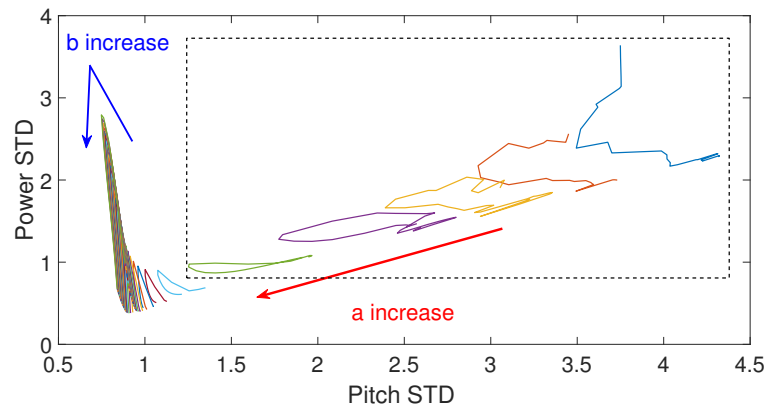

Figure 6: Generator Power STD - Blade Pitch STD Relationship (Each dots on one curve are with the same blade pitch weight)

Clearly, in Figure 6, lines with different generator torque weights do not have a strong regularity at the beginning, then show a strong correlation in both shape 


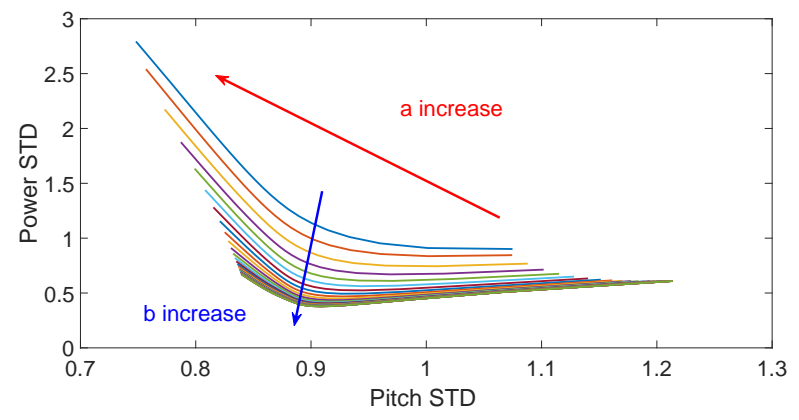

Figure 7: Effective Generator Power STD - Blade Pitch STD Relationship (Each dots on one curve are with the same generator torque weight)

and position when $a$ is greater than -5.2. As for each single line with the same generator torque weight, the generator power STD increases first and then decreases with the increase of $b$, which indicates that there always exists a smaller pitch STD on the later half of the curve than the first half once the generator power STD holds constant, and the optimal solution is always on the later half. Therefore, the part in which $a$ is less than -5.2 and every first half of each curves are removed to simplify the analysis, and the simplified data are plotted in Figure 7.

Different from Figure 6, all curves in Figure 7 are based on the generator torque weight. More specifically, each data point on one curve owns the same generator torque weight (the same $b$ ), and curves with different colors are responses with different generator torque weights. With the increase of blade pitch weight $a$, the blade pitch STD decreases on every curve. It can be concluded that, an infrequent blade pitch movement can be guaranteed by increasing the blade pitch weight when the generator torque weight holds constant, which is consistent with the definition of the cost function for controller design: to reduce the blade pitch movement by increasing the proportion of the blade pitch weight in the entire cost function. But the generator power STD shows a very interesting trend as gradually decreasing at first and then increasing. On the other hand, with the increase of generator torque weight $b$, curves gradually decrease in generator power STD and converge to an optimal boundary, which is the optimal boundary with the optimal generator torque weight $b$. However, the optimal boundary and optimal $b$ cannot be obtained, because the curves keep moving downward with the increase of $b$. The differences among the bottom curves still exist even it is hard to observe. Hence, we have done sufficient simulations (1680 groups) to make sure such a trend always continues, and a satisfactory generator torque weight is needed to keep $b$ from increasing to infinity. Since the curves are based on the generator torque weight, the number of points on each 
curve are the same. The differences among curves (curve difference) are obtained by the following equation:

$$
D_{k}=\sum_{i=1}^{M} \sqrt{\left(X_{k+1, i}-X_{k, i}\right)^{2}+\left(Y_{k+1, i}-Y_{k, i}\right)^{2}}, \quad k \in[1, N-1],
$$

where $X_{k, i}$ and $Y_{k, i}$ are the coordinates of the $i$-th data point on the $k$-th curve, $M$ is the number of point on each curve, and $N$ is the number of curves. The above equation calculates the Euclidean metric between two points with the same $a$ value, which is added up for one curve to further evaluate the difference between two nearby curves caused by the increase of generator torque weight. The curve difference of each $b$ is plotted in Figure 8.

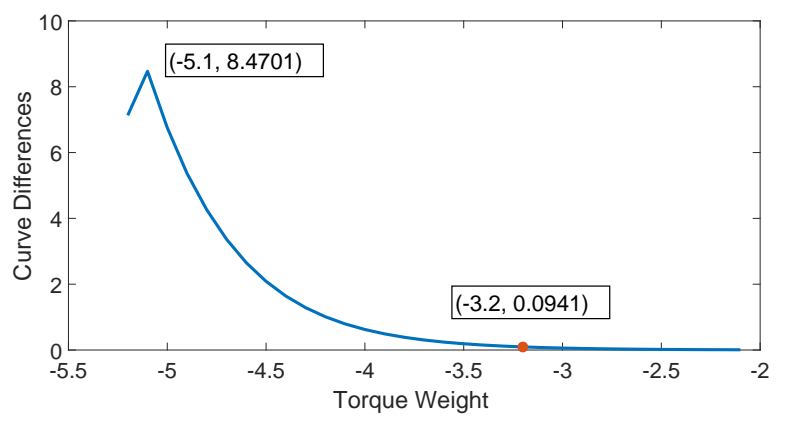

Figure 8: Curves Difference (Evaluate the difference between different generator torque weights.)

In Figure 8, the curve difference increases slightly in the beginning where $b$ equals to -5.2 , and reaches its maximum at -5.1 . Then it gradually reduces when $b$ continues to increase, and shows a smoothly declining trend. As it can be found that, the curve difference reduces less than 0.1 when $b$ equals to -3.2 , then changes to 0 gradually. Since the curve difference at this point is with an order of magnitude smaller than its maximum value, it can be approximated that there is no large difference in the rest curves after -3.2 . Hence, -3.2 is selected as the satisfactory $b$. The responses with different blade pitch weight $a$ when $b=-3.2$ are plotted in Figure 9.

As shown in Figure 9, the generator power STD keeps decreasing with the increase of $a$, reaches its minimum point when $a$ equals to 2.2 and turns to increase while the blade pitch movement STD continues to decrease. It implies that both the output quality and the load conditions are improved at first, after the minimum generator power STD point, the improvement of wind turbine load conditions is at the expense of increasing the instability of generator power output quality. On the other hand, the optimal load condition point (the minimum blade pitch movement 


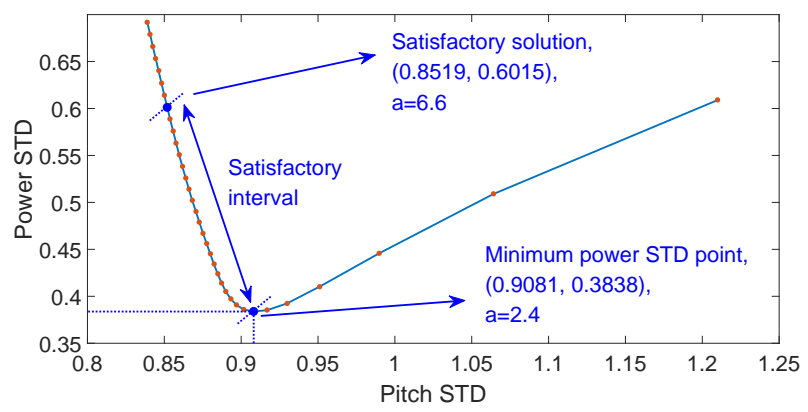

Figure 9: $b=-3.2$ (The responses with the same generator torque weight as $10^{-3.2}$, each dot represent one simulation result under different $a$ but the same $b$, the satisfactory interval bounded by minimum power STD point and satisfactory solution is marked in this figure.)

STD point) does not exist. It is also logically understandable that the increase of blade pitch weight in the cost function leads to a less frequent blade pitch action in a turbulent wind, so the generator power output fluctuates with the turbulence. The reduction of the blade pitch action will continue as $a$ increases, until the blade pitch angle is stabilized to a constant setting value. The above analysis shows that the increase of the pitch weight in the cost function can not be infinite. In contrast, the previous analysis shows that the increase of the generator torque weight can be infinite although numerically impossible. Between the optimal power output point and the infinite optimal blade pitch point, the improvement of any of the two targets is contradictory. All the weight combinations on this interval are the weak Pareto solutions and make this interval as the Pareto frontier. The satisfactory $a$ on the Pareto frontier should keep a balance between the generator power output and blade pitch actions. Herein, 6.6 is selected as the satisfactory $a$ to balance the considered optimization targets, for which the blade pitch STD is the most important variable to reduce loads, and the generator power output deteriorates a little but is still kept in an acceptable level. However, the satisfactory solution selection is still very subjective. Different solutions might be selected under different requirements, and such property makes the selected solution as a satisfactory one. Thus, a group of satisfactory weight combinations have been selected that $a=6.6$ and $b=-3.2$, and the region for $a \in[-6.6,-2.4]$ with $b=-3.2$ as the satisfactory interval. The comparison between the satisfactory solution and other control strategies will be discussed in the next section. 


\section{Wind-assessment-based Variable-weight MPC Strategy}

The satisfactory solution has been obtained through the previous analysis. In this section, the responses corresponding to two selected solutions are evaluated to illustrate the difference. A wind assessment-based variable-weight method is introduced to change the blade pitch weight in the model predictive controller to achieve better responses considering both generator power output and blade pitch movement.

\subsection{Selected Solutions Comparison}

As discussed in the above section, two solutions have been selected as the start and end boundary of the satisfactory interval, in which the solutions are with the same generator torque weight but different blade pitch weights. To analyze the difference between solutions, the selected optimal power solution and the satisfactory solution are compared as two examples to show the difference, which are simulated under a $15 \mathrm{~m} / \mathrm{s}$ mean speed turbulent wind condition with turbulence intensity 8 . The simulations are based on the FAST simulator developed by NREL (National Renewable Energy Laboratory). The tested wind is generated by TurbSim by following the IEC standard. The wind turbine basic characteristics include the generator power output, generator speed, generator torque conditions and the blade pitch actions as shown in Figure 10. In the figure, the blue curves show the responses of the satisfactory solution, while the orange ones show the responses of the optimal power solution.
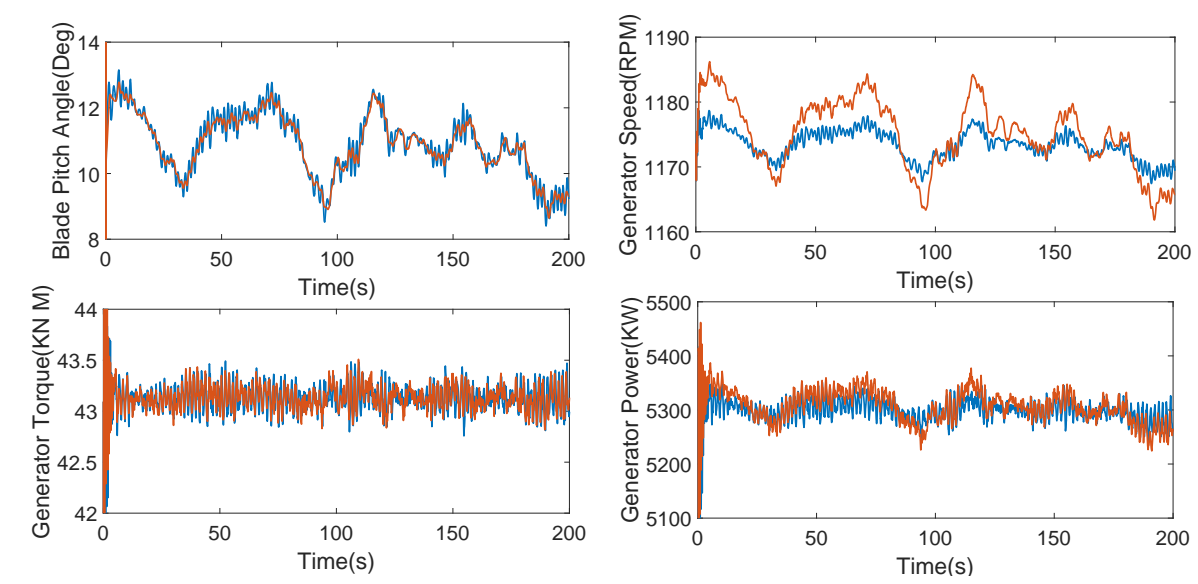

Figure 10: Selected Solution Comparison

As shown in Figure 10, the optimal power solution achieves a very stable generator power output. The turbulent wind reaches the general turbulence intensity and the power output is almost constant at the rated power $(5296.61 \mathrm{Kw})$ with only a 
few fluctuations. The same situation also occurs in the generator torque response. As to the generator speed, there is a slight fluctuation around the rated speed, which is not too large. Compared with the optimal power solution, the satisfactory solution maintains an acceptable situation. The generator torque response is basically consistent with the optimal power solution. The generator speed response and generator power output fluctuations are improved, which remains at an acceptable level. According to the standard value in Figure 9, the improvement of the satisfactory solution from the baseline controller is obvious. However, the blade pitch response on the top left in Figure 10 shows a different situation. The orange curve represents the blade pitch action under the satisfactory solution, which moves with the wind turbulent gradually and smoothly. The blue curve constantly oscillates around the orange curve frequently, which illustrates that for the optimal power solution, the corresponding blade pitch acts very frequently with the power oscillation caused by the wind turbulence. In other word, the optimal power solution makes a great sacrifice in the pitch action in order to ensure stable output of power. Such property is useful under a strong turbulence, but the frequency pitch actions do not help so much when the turbulence is not so extreme but will cause the stress of the blade to gather and increase the fatigue loads.

\subsection{Wind-sssessment-based Variable-weight Strategy}

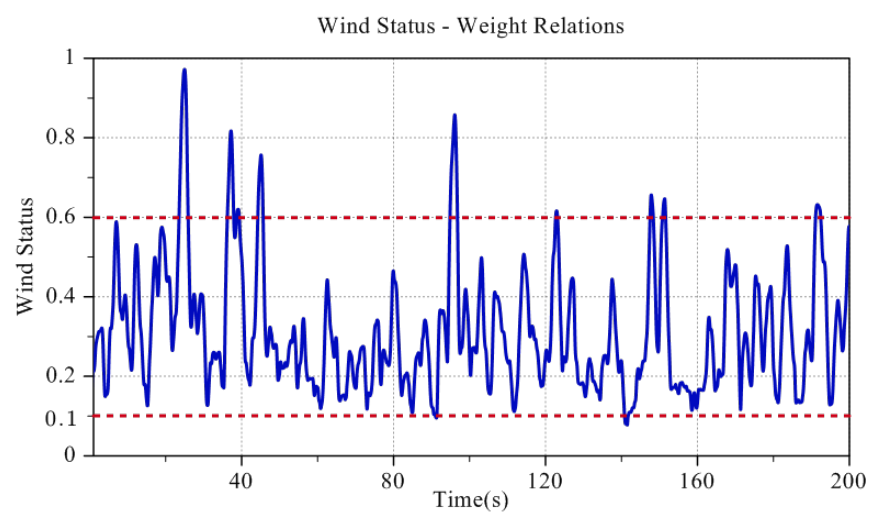

Figure 11: Wind Speed Status

Since the wind turbulence is not static, it is necessary to select the appropriate controller-weight setting under different turbulence intensity. A power-stability based controller-weight set is helpful to achieve the stable power output through the frequent blade pitch action under high turbulent conditions. The comprehensive 
weight set for both power output stability and pitch movement is useful to keep the output power stable, while reducing unnecessary pitch actions if the turbulence is not so strong. To assess the wind turbulence intensity, the measured wind first passes through a low-pass filter to filter out wind speed noise with one second resolution. Then the filtered wind is calculated into standard deviation every 5 seconds through the sliding window method as the assessed wind speed status. The simulated wind speed status is plotted in Figure 11, for which the wind status fluctuates mostly between 0.1 and 0.6 and beyond this interval occasionally. Therefore, the variable weight region is selected as the interval between 0.1 and 0.6. Two dead zones are set beyond the variable weight interval, in which the blade pitch weight is constant for the optimal power solution when the wind status over 0.6, and constant for the satisfactory solution when below 0.1 . In the variable weight region, the blade pitch weight is set as the linear interpolation of the front and back dead zone boundaries. This strategy can be graphically represented in Figure 12.

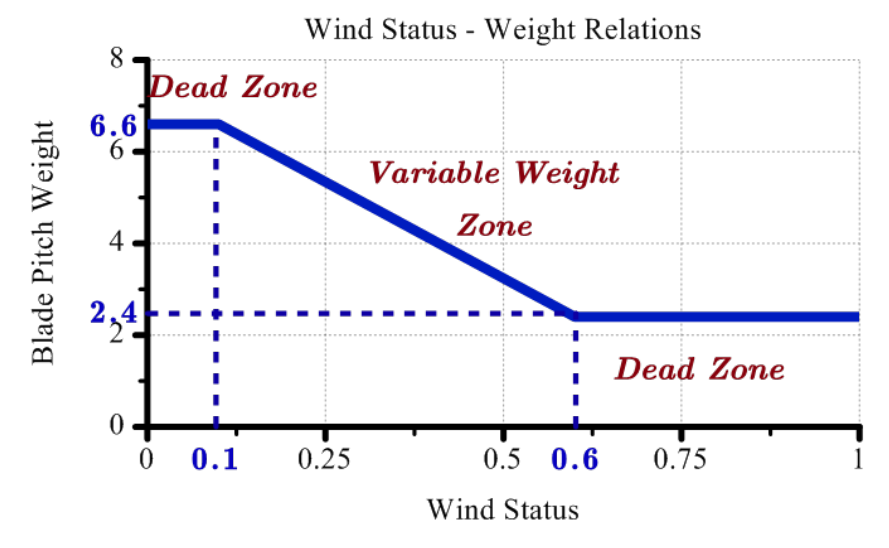

Figure 12: Wind Status - Blade Pitch Weight Relations

\subsection{Effectiveness Evaluation}

To verify the effectiveness of the proposed variable-weight MPC strategy, it is simulated based on the same environment as the previous simulations. As follows, this strategy is compared with the satisfactory solution, and the widely used blade-pitchgain-scheduling PI control by recalling the baseline controller provided in FAST. The detail definition about the gain scheduling PI controller can be referred to [29].

In Figure 13, the blue curves stand for the proposed variable-weight MPC strategy, while the orange curves represent the satisfactory solution. It can be found that, the proposed strategy achieves more stable responses for both generator power 

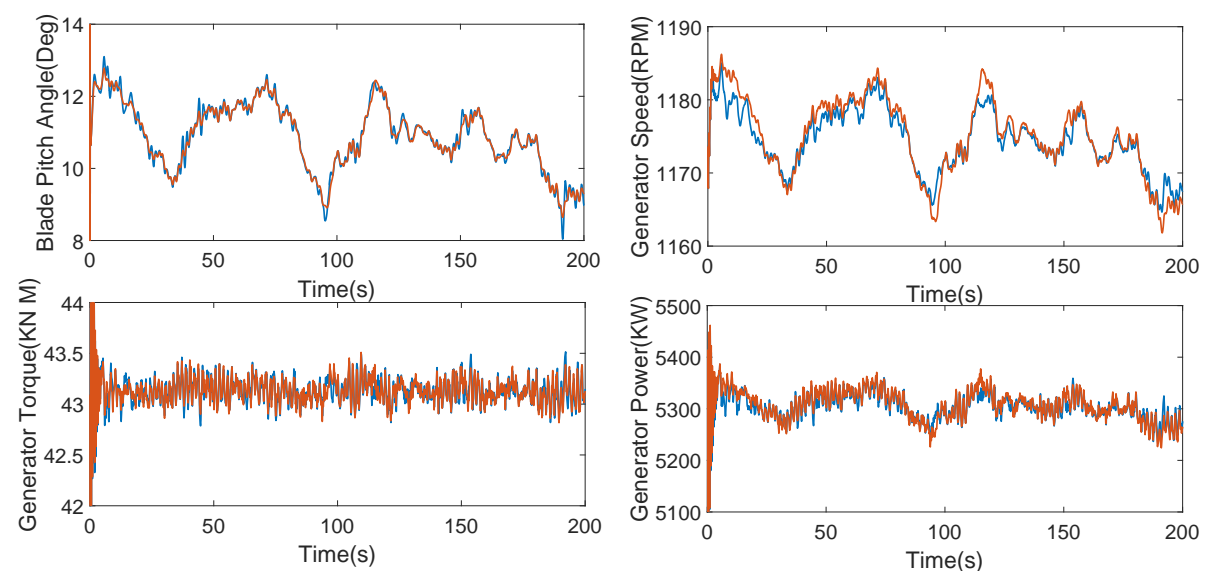

Figure 13: Effectiveness evaluation: Comparison between the variable weight strategy and the satisfactory solution

output and generator speed than the satisfactory solution. Especially, the relatively obvious part of the fluctuation in the satisfactory solution has been effectively reduced with the proposed strategy. As to the blade pitch actions, frequent variable pitch actions are introduced to stabilize power and speed when the turbulence is strong. The pitch angle action of the proposed strategy is basically the same as the satisfactory solution most of the time. In another word, the frequent pitch actions are used only if necessary.
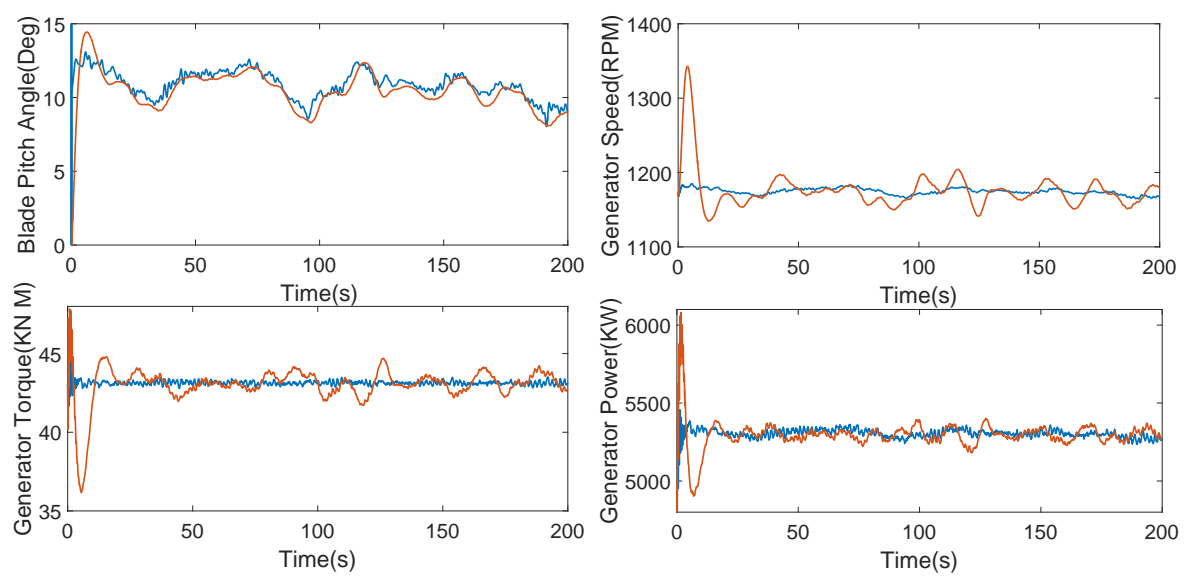

Figure 14: Effectiveness evaluation: Comparison between the variable weight strategy and the baseline controller

In Figure 14, the variable-weight MPC is compared with the baseline controller. 
The blue curves are the proposed controller and the orange curves are the baseline one. Clearly, the generator speed, generator torque and generator power output with the variable-weight MPC are much more stable compared with the baseline controller, which are nearly with no fluctuations. The blade pitch action curves have the same trend but the variable-weight one moves more flexibly than the baseline controlled one under turbulence. In order to ensure that the increase of pitch sensitivity does not lead to an increase in wind turbine loads, the out-of-plane bending moment on the blade root (RootMyc) and the low-speed shaft thrust force on the drive-shaft (LSShftFxs) are selected as the most important load parameters to evaluate the turbine load conditions. Both of the mean value and max value are calculated and standardized based on the corresponding parameter under the baseline controller, which are shown in the histogram form in Figure 15.

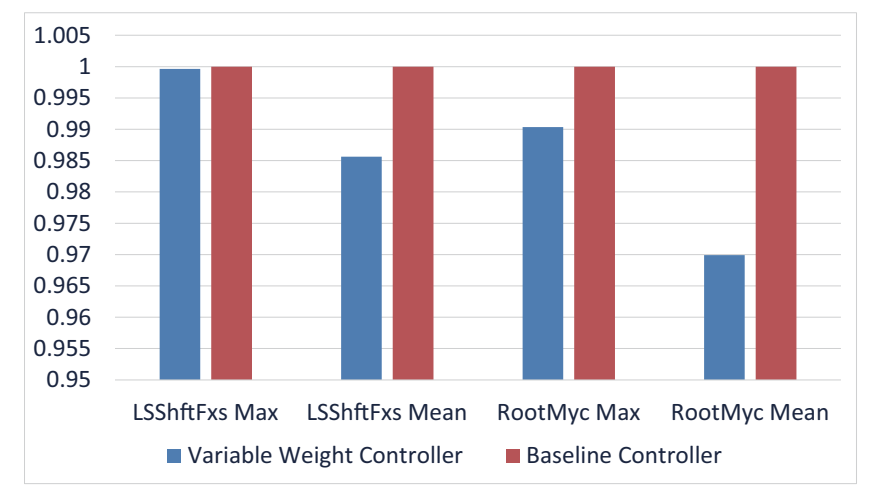

Figure 15: Loads analysis : Comparison between the variable weight strategy and the baseline controller. Load parameters with the variable weight MPC controller are in blue, and corresponding parameters with the baseline controller are in red

It can be found that, the variable-weight MPC has a better load condition in both the maximum value and mean value of the analyzed load parameters than the baseline controller. The analysis confirms that the increase in blade pitch sensitivity does not result in a rise in load. The variable-weight MPC can achieve better responses in all concerned objectives, including the turbine working conditions, load conditions and power output. The above analysis also indicates that, although the baseline controller can achieve good control effects, there still exists plenty of improvement room from the baseline controller to the Pareto frontier.

\section{MPC Tuning Suggestions}

In addition to the proposed variable-weight MPC, the above analysis results can also be used in other cases for the optimization of wind turbine operation. 


\subsection{Quantified Satisfactory Solution Selection}

The solutions are defined as feasible solutions by considering their improvements on both the lower blade pitch STD and the generator power STD compared with the baseline controller. The feasible solutions can be included in an arc boundary with $(1,1)$ point as the origin. The origin point is the equivalent baseline controller, since all the response parameters are unitarily based on the baseline controller. In such a way, all feasible solutions can be described by a combination of two parameters: the distance from the origin $\sqrt{(x-1)^{2}+(y-1)^{2}}$, and the ratio of the coordinates difference relative to the origin $\frac{y-1}{x-1}$. The first parameter can be regarded as the improvement of this feasible solution relative to the baseline controller, and the second one represents the proportion of the improvement in power stability and loads conditions. This provides a quantitative solution to the selection of the satisfactory solution: set the overall improvement ratio from the baseline controller and the improvement proportion between two objectives, then one target point can be plotted in the previous delimited arc. If less than the target point within both coordinates, the feasible solutions will be considered as the feasible set under current conditions to ensure that the improvement is not less than the set target. Then try to find the feasible solution closest to this optimized target as the satisfactory solution. The improvement ratio of the satisfactory solution selection is required to be within a boundary between the equivalent baseline controller and the envelope. The schematic diagram of this strategy is shown as in Figure 16.

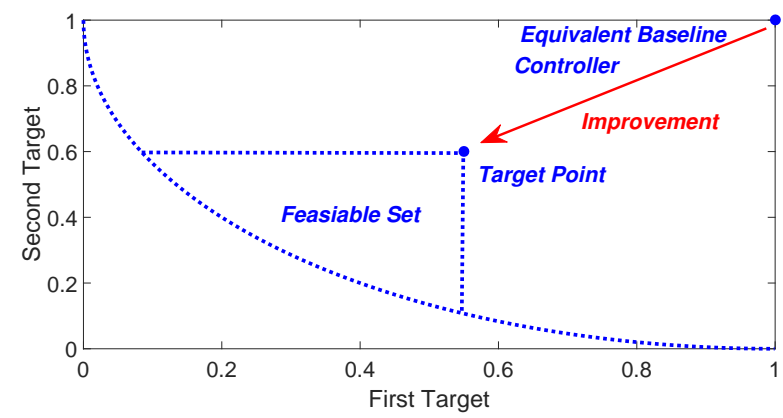

Figure 16: Quantified Satisfactory Solution Selection Schematic

It should be noticed that, the correlation between the blade pitch STD and the blade/shaft loads summarized in the above sections is a qualitative correlation. Although the correlation properties of the response data can be fitted as a linear or polynomial relationship, such description is still not precise and comprehensive enough. The proposed strategy aims to simplify the analysis process, and provide a simplified but effective analysis method for the controller tuning. 


\subsection{Controller Tuning Suggestion}

Based on the above discussion, a controller tuning suggestion can be summarized and applied in the wind turbine MPC tuning process. As shown in Figure 7, the increase of the generator torque weight $b$ always improves the generator power output and loads conditions, although the improvement is not significant enough when $b$ is large to access in the inert interval shown in Figure 17. The blade pitch weight $a$ should reach a Pareto frontier to make sure the solution on the optimal frontier and then to choose a satisfactory one to balance the generator power output quality and blade pitch actions. During the MPC tuning process, the generator torque weight can be set with a big value to ensure the controller working properly to protect the system stability, and the blade pitch weight $a$ can start with a small value that the generator power output is the main variable considered at first. The blade pitch weight $a$ continues increasing to find the minimum power STD point. After the optimal power point, the increase of $a$ maintains the response on the Pareto frontier and begins to improve the load conditions. The blade pitch action frequency keeps decreasing but increasing the output power instability, until the blade pitch weight $a$ stops at a satisfactory point, which achieves a balance between optimization targets before the output quality deteriorates seriously.

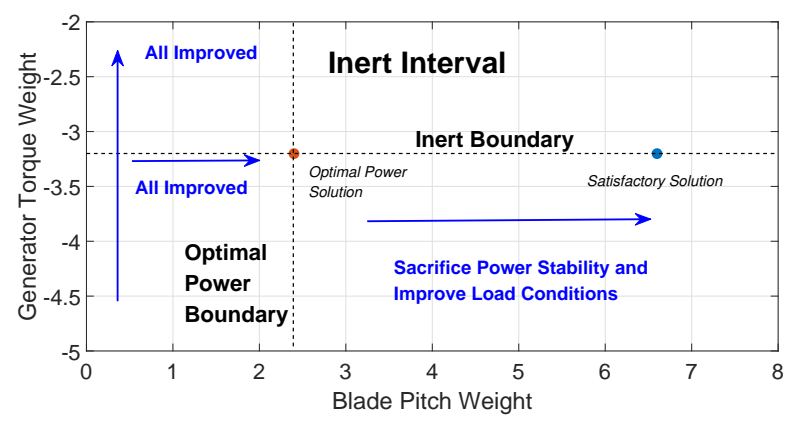

Figure 17: Wind Turbine Model Predictive Controller Tuning Advices

\subsection{Applied in Other Cases}

It is worth mentioning that the simulated wind turbine is an onshore wind turbine. From the viewpoint of control design, the most difference between the offshore and onshore wind turbine is that, the offshore one has to use the constant torque strategy instead of the constant power strategy above the rated wind speed. Instead of stabilizing the generator power output, the offshore wind turbine has to maintain a lower level of frequent pitch actions and generator torque fluctuations to avoid the 
high loads conditions caused by mechanical resonance. In such a case, the proposed qualitative correlation from the blade pitch STD with blade/shaft load maximum value could be applied in the offshore wind turbine control design to consider the mechanical loads. Although the test and analysis are focused on the 5-MW NREL wind turbine, such parameter correlation is universally applicable to different types of HAWTs.

\section{Conclusion}

This paper proposes a variable-weight MPC strategy to optimize the mechanical loads and power of wind energy conversion system, in which the pitch/torque participation coordination are adjusted to operate appropriately. Based on the MPC framework, the weight matrix is constructed and tuned in detail, which reflects the pitch/torque participation and affects the operation effects significantly. For better load reductions, the correlation relationship from the drive shaft and blade load parameters to the blade pitch movement STD is analyzed and established. Both generator power output quality and loads conditions are considered more closely to optimize the pitch/torque coordination based on the Pareto optimization theory. A group of solutions on the Pareto frontier including the optimal power solution is selected as the satisfactory interval. Based on the satisfactory interval and the wind assessment method, a variable-weight strategy is proposed to optimize the WECS operations adaptively. The comparisons between the proposed strategy and the gain scheduling PI one show the effectiveness and improvement.

\section{Appendix A. Abbreviations}

$\begin{array}{ll}\text { FAST } & \text { Fatigue, Aerodynamics, Structures, and Turbulence } \\ \text { LSShiftFxs } & \text { Root bending moments in } x_{s} \text { axes } \\ \text { LSShiftFys } & \text { Root bending moments in } y_{s} \text { axes } \\ \text { LSShiftFzs } & \text { Root bending moments in } z_{s} \text { axes } \\ \text { LSS } & \text { Low speed shaft } \\ \text { MPC } & \text { Model predictive control } \\ \text { MW } & \text { Megawatt } \\ \text { NREL } & \text { National Renewable Energy Laboratory } \\ \text { PI } & \text { Proportional-Integral } \\ \text { QP } & \text { Quadratic programming } \\ \text { RootMxc } & \text { Root bending moments in } x_{c} \text { axes } \\ \text { RootMyc } & \text { Root bending moments in } y_{c} \text { axes }\end{array}$


RootMzc Root bending moments in $z_{c}$ axes

STD Standard deviation

TRIZ Theory of inventive problem solution

WECS Wind turbine generator system

\section{Appendix B. Symbols}

$\begin{array}{ll}a & \text { The blade pitch angle weight } \\ A & \text { Discrete model state matrix } \\ A_{c} & \text { Continues model state matrix } \\ b & \text { The generator torque weight } \\ B & \text { Discrete model input matrix } \\ B_{c} & \text { Continues model input matrix } \\ C_{p} & \text { Wind power coefficient } \\ D_{k} & \text { Curve difference between the } k \text {-th and } k+1 \text {-th curve } \\ F_{x} & \text { Predictive model state matrix } \\ G_{x} & \text { Predictive model input matrix } \\ P_{a} & \text { Aerodynamic power } \\ Q_{x} & \text { State weight matrix } \\ R_{u} & \text { Control weight matrix } \\ R & \text { Rotor radius } \\ T_{e m}^{r e f} & \text { Referenced generator torque } \\ T_{e m}^{R a t e d} & \text { Rated generator torque } \\ U_{(k)} & \text { Predicted control parameters } \\ X(k) & \text { Predicted state parameters } \\ X_{k, i} & \text { x-axis coordinate of the } i \text {-th data point on the } k \text {-th curve } \\ Y_{k, i} & y \text {-axis coordinate of the } i \text {-th data point on the } k \text {-th curve } \\ \beta & \text { Blade pitch angle } \\ \beta_{\text {ref }} & \text { Referenced blade pitch angle } \\ \delta & \text { Shaft equivalently torsional angle } \\ \lambda & \text { Tip speed ratio } \\ \rho & \text { Air density } \\ v & \text { Wind Speed } \\ \psi_{\text {Self }} & \text { Parameters under the model predictive controller } \\ \psi_{B C} & \text { Parameters under the baseline controller. } \\ \omega_{g} & \text { Generator speed } \\ \omega_{g_{R a t e d}} & \text { Rated generator speed } \\ \omega_{r} & \text { Rotor speed } \\ \omega_{r_{R a t e d}} & \text { Rated rotor speed } \\ & \end{array}$


[1] GWEC, Global wind report 2017, http://gwec.net/publications/global-windreport-2/, [Online] (April 2018).

[2] D. Kumar, K. Chatterjee, A review of conventional and advanced mppt algorithms for wind energy systems, Renewable and sustainable energy reviews 55 (2016) 957-970.

[3] L. González, E. Figueres, G. Garcerá, O. Carranza, Maximum-power-point tracking with reduced mechanical stress applied to wind-energy-conversionsystems, Applied Energy 87 (7) (2010) 2304-2312.

[4] B. Govind, Increasing the operational capability of a horizontal axis wind turbine by its integration with a vertical axis wind turbine, Applied Energy 199 (2017) 479-494.

[5] A. R. Jha, Wind turbine technology, CRC press, 2010.

[6] T. Senjyu, R. Sakamoto, N. Urasaki, T. Funabashi, H. Fujita, H. Sekine, Output power leveling of wind turbine generator for all operating regions by pitch angle control, IEEE Transactions on Energy conversion 21 (2) (2006) 467-475.

[7] X. Xing, H. Meng, L. Xie, L. Yue, L. Zhong-wei, Switching performance improvement based on model predictive control for wind turbine covering whole wind speed range, IEEE Transactions on Sustainable Energy (2018) 11doi:10.1109/TSTE.2018.2833634.

[8] M. Yin, Z. Yang, Y. Xu, J. Liu, L. Zhou, Y. Zou, Aerodynamic optimization for variable-speed wind turbines based on wind energy capture efficiency, Applied Energy 221 (2018) 508-521.

[9] Z. Lin, Z. Chen, Q. Wu, S. Yang, H. Meng, Coordinated pitch \& torque control of large-scale wind turbine based on pareto efficiency analysis , Energy 147 (2018) $812-825$.

[10] M. L. Corradini, G. Ippoliti, G. Orlando, An observer-based blade-pitch controller of wind turbines in high wind speeds, Control Engineering Practice 58 (2017) 186-192.

[11] C. A. Evangelista, A. Pisano, P. Puleston, E. Usai, Receding horizon adaptive second-order sliding mode control for doubly-fed induction generator based wind turbine, IEEE Transactions on Control Systems Technology 25 (1) (2017) 73-84. 
[12] X.-x. Yin, Y.-g. Lin, W. Li, Y.-j. Gu, P.-f. Lei, H.-w. Liu, Adaptive back-stepping pitch angle control for wind turbine based on a new electro-hydraulic pitch system, International Journal of Control 88 (11) (2015) 2316-2326.

[13] B. Yang, T. Yu, H. Shu, J. Dong, L. Jiang, Robust sliding-mode control of wind energy conversion systems for optimal power extraction via nonlinear perturbation observers, Applied Energy 210 (2018) 711-723.

[14] Z. Lin, J. Liu, Y. Niu, Dynamic response regulation of non-linear feedback linearised wind turbine using a two-mass model, IET Control Theory \& Applications 11 (6) (2017) 816-826.

[15] R. Ata, Artificial neural networks applications in wind energy systems: a review, Renewable \& Sustainable Energy Reviews 49 (2015) 534-562.

[16] V. Nikolić, S. Sajjadi, D. Petković, S. Shamshirband, Ž. Ćojbašić, L. Y. Por, Design and state of art of innovative wind turbine systems, Renewable \& Sustainable Energy Reviews 61 (2016) 258-265.

[17] D. Petković, N. T. Pavlović, Ž. Ćojbašić, Wind farm efficiency by adaptive neuro-fuzzy strategy, International Journal of Electrical Power \& Energy Systems 81 (2016) 215-221.

[18] D. Petković, V. Nikolić, V. V. Mitić, L. Kocić, Estimation of fractal representation of wind speed fluctuation by artificial neural network with different training algorothms, Flow Measurement \& Instrumentation 54 (2017) 172-176.

[19] D. Petković, Ž. Ćojbašić, V. Nikolić, S. Shamshirband, M. L. M. Kiah, N. B. Anuar, A. W. A. Wahab, Adaptive neuro-fuzzy maximal power extraction of wind turbine withcontinuously variable transmission, Energy 64 (1) (2014) 868874 .

[20] D. Song, X. Fan, J. Yang, A. Liu, S. Chen, Y. H. Joo, Power extraction efficiency optimization of horizontal-axis wind turbines through optimizing control parameters of yaw control systems using an intelligent method, Applied Energy 224 (2018) 267-279.

[21] Z. Lin, J. Liu, W. Zhang, Y. Niu, Regional pole placement of wind turbine generator system via a markovian approach, IET Control Theory \& Applications 10 (15) (2016) 1771-1781. 
[22] R. Melício, V. M. F. Mendes, J. P. d. S. Catalão, Transient analysis of variablespeed wind turbines at wind speed disturbances and a pitch control malfunction, Applied Energy 88 (4) (2011) 1322-1330.

[23] Y. Cui, Z. Geng, Q. Zhu, Y. Han, Multi-objective optimization methods and application in energy saving, Energy 125 (2017) 681-704.

[24] Y. W. Pratama, W. W. Purwanto, T. Tezuka, B. C. Mclellan, D. Hartono, A. Hidayatno, Y. Daud, Multi-objective optimization of a multiregional electricity system in an archipelagic state: The role of renewable energy in energy system sustainability, Renewable \& Sustainable Energy Reviews 77 (2017) 423439.

[25] P. F. Odgaard, L. F. S. Larsen, R. Wisniewski, T. G. Hovgaard, On using pareto optimality to tune a linear model predictive controller for wind turbines, Renewable Energy 87 (2016) $884-891$.

[26] P. F. Odgaard, On usage of pareto curves to select wind turbine controller tunings to the wind turbulence level, in: Control Conference (ECC), 2015 European, IEEE, 2015, pp. 1534-1539.

[27] A. Rezaeiha, I. Kalkman, B. Blocken, Effect of pitch angle on power performance and aerodynamics of a vertical axis wind turbine, Applied Energy 197 (2017) $132-150$.

[28] S. Nourdine, H. Camblong, I. Vechiu, G. Tapia, Comparison of wind turbine lqg controllers using individual pitch control to alleviate fatigue loads, in: IEEE International Conference on Control and Automation, 2011, pp. 1502-1507.

[29] J. Jonkman, S. Butterfield, W. Musial, G. Scott, Definition of a 5-mw reference wind turbine for offshore system development, Tech. rep., National Renewable Energy Lab.(NREL), Golden, CO (United States) (2009).

[30] J. M. Jonkman, M. L. Buhl Jr, et al., Fast users guide, National Renewable Energy Laboratory, Golden, CO, Technical Report No. NREL/EL-500-38230.

[31] A. Mosek, The mosek optimization toolbox for matlab manual (2015).

[32] S. Boyd, L. Vandenberghe, Convex optimization, Cambridge university press, 2004. 